\title{
A Multiagent System for Web-Based Risk Management in Small and Medium Business
}

\author{
Juan F. De Paz, Javier Bajo, M. Lourdes Borrajo, and Juan M. Corchado
}

\begin{abstract}
Business Intelligence has gained relevance during the last years to improve business decision making. However, there is still a growing need of developing innovative tools that can help small to medium sized enterprises to predict risky situations and manage inefficient activities. This article present a multiagent system especially conceived to detect risky situations and provide recommendations to the internal auditors of SMEs. The core of the multiagent system is a type of agent with advanced capacities for reasoning to make predictions based on previous experiences. This agent type is used to implement an evaluator agent specialized in detect risky situations and an advisor agent aimed at providing decision support facilities. Both agents incorporate innovative techniques in the stages of the CBR system. An initial prototype was developed and the results obtained related to small and medium enterprises in a real scenario are presented.
\end{abstract}

Keywords: Hybrid neural intelligent system, CBR, MAS, Business Intelligence, business risk prediction.

\section{Introduction}

Nowadays, the organization systems employed in enterprises are increasing in complexity. In the present financial context, it is increasilly relevant to provide innovative tools and decision support systems that can help the small-medium

\author{
Javier Bajo \\ Facultad de Informática. Universidad Pontificia de Salamanca Compañía 5, \\ 37002, Salamanca, Spain \\ e-mail: jbajope@upsa.es \\ M. Lourdes Borrajo \\ Dept. Informática, University of Vigo, Edificio Politécnico, \\ Campus As Lagoas s/n, Ourense, 32004, Spain \\ e-mail: Iborrajo@uvigo.es \\ Juan F. De Paz · Juan M. Corchado \\ Departamento Informática y Automática. University of Salamanca, Plaza de la Merced s/n, \\ 37008, Salamanca, Spain \\ e-mail: \{fcofds, corchado\}@usal.es
}


enterprises (SMEs) to improve their functioning (Khashman 2009, Li and Sun in press, Li et al. in press, Sun and Li 2009a, Sun and Li 2009b). These tools and methods can contribute to improve the existing business control mechanisms, reducing the risk by predicting undesiderable situations and providing recommendations based on previous experiences (Chi-Jie 2009, Li and Su 2008, Sun and Li 2008a, Sun and Li 2008b).

The processes carried out inside a company are grouped into functional areas (Corchado et al. 2000) denominated "Functions". A Function is a group of coordinated and related activities that are systematically and iteratively carried out during the process of reaching the company's objectives (Li and Sun 2009). The functions that are usually carried out within a company, as studied within the framework of this research, are: Purchases, Cash Management, Sales, Information Technology, Fixed Assets Management, Compliance to Legal Norms and Human Resources. Each one of these functions is broken down into a series of activities. For example, the Information Technology function is divided into the following activities: Computer Plan Development, Study of Systems, Installation of Systems, Treatment of Information Flows, and Security Management.

This article propose an innovative approach, based on multiagent systems (Bajo et al. 2009), to propose a model for risk management and prediction in SMEs. Multiagent systems are the most prevalent solution to construct Artificial Intelligence distributed systems. Agents are computational entities that can be characterized through their capacities in areas such as autonomy, reactivity, pro-activity, social abilities, reasoning, learning and mobility (Bajo et al. 2009). These capacities make the multi-agent systems very appropriate for constructing intelligent environments. An agent can act as an interface between the user and the rest of the elements of the intelligent environment. Moreover, intelligent agents can incorporate advanced artificial intelligence models to predict risky situations.

The article is structured as follows: the next section briefly introduces the problem that motivates this research. Section 3 presents the multi-agent system for managing small and medium enterprises. Section 4 presents the results obtained after testing the system and the conclusions of this study.

\title{
2 Application of Business Web Intelligence to Enterprise Risk Assessment and Management
}

\begin{abstract}
"Risk Management" is a broad term for the business discipline that protects the assets and profits of an organization by reducing the potential for loss before it occurs, mitigating the impact of a loss if it occurs, and executing a swift recovery after a loss occurs. It involves a series of steps that include risk identification, the measurement and evaluation of exposures, exposure reduction or elimination, risk reporting, and risk transfer and/or financing for losses that may occur. All organizations practice risk management in multiple forms, depending on the exposure being addressed (Calderon and Cheh 2002, Risk and Insurance Management Society 2008).
\end{abstract}


The economic environment has increased the pressure on all companies to address risk at the highest levels of the organization. Companies with a strategic approach to risk management use more tools and have more structured and frequent reporting on risk management than do firms with other approaches. As such, they are in a better position to ensure that risk management provides relevant and applicable information that meets the needs of the organization and executive team. But no matter what an organization's approach is, the tools used must be backed up by solid, actionable reporting addressed (Calderon and Cheh 2002, Risk and Insurance Management Society 2008). It's not always necessary for the risk managers to be conducting their own studies for their voices to be heard. Forging a strong relationship with internal auditors and other departments can allow risk practitioners to supplement their reports with the risk manager's own analysis (Colbert 1995).

Enterprise Risk Management (ERM) is defined as "a process, effected by an entity's board of directors, management and other personnel, applied in strategysetting and across the enterprise, designed to identify potential events that may affect the entity, and manage risk to be within its risk appetite, to provide reasonable assurance regarding the achievement of entity objectives." (Committee of Sponsoring Organizations of the Treadway Commission -COSO 2009).

The managing of risks and uncertainties is central to the survival and performance of organizations. Enterprise risk management (ERM) is an emerging approach to managing risks across different business functions in an organization that represents a paradigm shift from specialized, silo-ed approaches in managing specific risks (Ding et al. 2008, Huang et al. 2008, Li and Sun 2009, Lin et al. 2009, Ramamoorti et al. 1999). This paper provides a web intelligent model to Enterprise risk assessment, which will subsequently lead to better organizational performance.

\section{Multiagent Web System for Risk Management}

Agent and multi-agent systems (SMEs) have become increasily relevant during the last decades and have gained relevance in different areas (Bajo et al. 2009, Sun and Li 2008).

In this article we propose a distributed approach where the components of a SME are modeled as intelligent agents that collaborate to create models that can evolve over the time and adapt to the changing conditions of the environment. The multiagent system provides a web system interface to facilitate the remote interaction with the human users involved in the risk management process.

Thus, making possible to detect risky situations for the SMEs and providing suggestions and recommendations that can help to avoid possible undesiderable situations. The agents in the system allow the users to access the system through distributed applications, which run on different types of devices and interfaces (e.g. computers, cell phones, PDA). Figure 1 shows the basic schema of the proposed architecture, where all requests and responses are handled by the agents in the platform. 


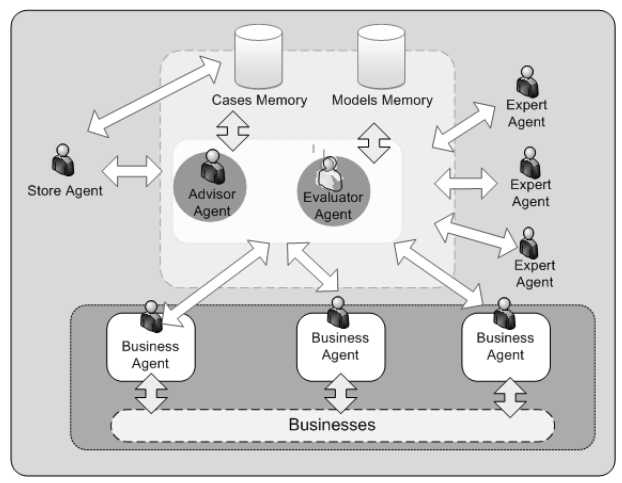

Fig. 1 Crisis multiagent architecture basic schema.

There are different kinds of agents in the architecture, each one with specific roles, capabilities and characteristics:

- Business Agent. This agent was assigned for each enterprise in order to collect new data and allow consultations. The enterprise can interact with the system by means of this agent, introducing information and receiving predictions.

- Evaluator Agent. It is responsible for the evaluation and predictions of potential risky situations.

- Advisor agent. The objective of this agent is to carry out recommendations to help the internal auditor decide which actions to take in order to improve the company's internal and external processes.

- Expert Agent. This agent helps the auditors and enterprise control experts that collaborate in the project to provide information and feedback to the multiagent system. These experts generate prototypical cases from their experience and they receive assistance in developing the Store agent case-base.

- Store Agent. This agent has a memory that has been fed with cases constructed with information provided by the enterprise (through its agent) and with prototypical cases identified by 34 enterprises control experts, using personal agents who have collaborated and supervised the developed model.

The core of the multiagent system are the evaluator and advisor agents, that incorporate new techniques to analyze the data from enterprises, extract the relevant information, and detect possible failures or inefficiencies in the operation processes.

The evaluator and advisor agents are CBR-BDI agents (Corchado and Laza 2003 ) that make use to past experiences to resolve new problems. As such, it is perfectly suited for solving the problem at hand. In addition, CBR (Case-Based Reasoning) makes it possible to incorporate the various stages of expression analysis into the reasoning cycle of the CBR (Kolodner 1993), thus facilitating the creation of strategies similar to the processes followed in small and medium enterprises.

On one hand the evaluator agent is specialized in detecting risky situations. The recovery of information from previous experiences simplifies the prediction 
process by detecting and eliminating relevant and irrelevant patterns detected in previous analyses. The retrieve phase of the hybrid neural intelligent system incorporates a novel Expectation Maximization clustering technique (Dellaert 2002). The reuse stage incorporates an innovative mixture of experts that makes use of multilayer perceptron, support vector regression and radial basis function neural network. The revise and retain stages implement a decision support system for experts. Moreover, the knowledge obtained during the prediction process is of great importance for subsequent predictions. On the other hand, the advisor agent is specialized in providing recommendations to avoid risky situations and improve the overall functioning of the SME. The retrieve phase recovers similar cases and their corresponding solutions. The reuse phase incorporates a novel approach based on decision trees and probabilistic gain functions to ponder efficient and inefficient tasks. The revise and retain stages also implement a decision support system for experts. There are various artificial intelligence techniques such as artificial neural networks (Bianchia et al. 2007, Sawa and Ohno-Machado 2003), Bayesian networks (Baladandayuthapani et al. 2005), and fuzzy logic (Avogadri and Valentini 2009) which have been applied to business failure prediction. While these techniques can be applied to failure detection and prediction, the knowledge obtained cannot be incorporated into successive tests and included in subsequent analyses. The approach presented in this article is an evolution of our previous works and proposes an innovative perspective (Borrajo et al. 2005, Corchado et al. 2005). The new approach proposes a multiagent system to model the organizational structure of a SME. Moreover, the core of the system is a CBR-BDI agent type with the ability to adapt to the changes in the environment. In (Borrajo et al. 2005) we presented a system composed of two case-based reasoning systems to detect the associate risk in the activities of SMEs in the textile sector and generate recommendations to improve the erroneous processes. In (Corchado et al. 2005) we presented a decision support tool based on a case-based reasoning system that automates the internal control processes of a SME. The new approach proposes new methods for the retrieval stage of the CBR systems, as the Expectation Maximization clustering, that notably improves the case's recovery reducing the final quantity of cases stored and making it easier to recover the most similar cases to the problem introduced. Moreover, the approach proposes very innovative reuse mechanisms, based on mixture of experts and probabilistic decision trees.

\section{Results and Conclusions}

A case study aimed at providing innovative web business intelligence tools for the management of SMEs was carried out in the Castilla y León region, in Spain. The experiment consisted on the construction of the initial prototype of memory of cases and then in predicting potential risky situations for the enterprises taken into considerations and providing recommendations. The case study presented in this work was oriented to detect possible risky situations in SMEs, taken into account the crisis that affects the market. A multiagent system was implemented and 22 SMEs participated in the experiment and were assigned a personal business agent. The enterprises were situated in different sectors and located in the Spanish region 
of Castilla y León. The economic context is the same for all the SMEs. The system was tested during 24 months, from January 2008 to January 2010, tuned and improved taking into account the experience acquired using a total of 238 cases. The evolution of the enterprise is monitored through its internal activities and the predictions are made based on the previous experiences and on the status of the market (the possible crisis that affect the market). To provide information about the Enterprise, the experts have to complete a survey.

To validate the overall functioning of the system it was necessary to individually evaluate the Evaluator and Advisor agents. These agents provide predictions on the performance of the activities and detect those tasks that can be improved for each activity in order to get an overall improvement of the activity. To validate the performance of the Evaluator agent, an estimation of the efficiency of the predictions provided by the Evaluator agent was carried out. To evaluate the significance of the different techniques integrated within the Evaluator agent, a cross validation was established, following the Dietterich's 5x2- Cross-Validation Paired t-Test algorithm (Dietterich 1998). The value 5 in the algorithm represents the number of replications of the training process and value 2 is the number of sets in which the global set is divided. Thus, for each of the techniques, the global dataset $\mathrm{S}$ was divided into two groups $S_{1}$ and $S_{2}$ as follows: $S=S_{1} \cup S_{2}$ y $S_{1} \cap S_{2}=\phi$. Then, the learning and estimation processes were carried out. This process was repeated 5 times and had the following steps: the system was trained using $S_{1}$ and then it was used to classify $S_{1}$ y $S_{2}$. In a second step, the system was trained using $S_{2}$ and then it was used to classify $S_{1}$ y $S_{2}$. The results obtained by the evaluator agent using the mixture of experts were compared to the results obtained using an individual RBF and an individual MLP to the same dataset and the same 5x2- CrossValidation process. Table 1 shows the error rate obtained for each of the techniques, using the test in each of the 5 repetitions. As can be seen in Table 1, the estimated error was lower for the Evaluator agent than for the rest of the evaluated techniques.

Table 1 Absolute error for the estimation of the status of the activities.

\begin{tabular}{lcccccccccc}
\hline Method & $\mathrm{S}_{2}$ & $\mathrm{~S}_{1}$ & $\mathrm{~S}_{2}$ & $\mathrm{~S}_{1}$ & $\mathrm{~S}_{2}$ & $\mathrm{~S}_{1}$ & $\mathrm{~S}_{2}$ & $\mathrm{~S}_{1}$ & $\mathrm{~S}_{2}$ & $\mathrm{~S}_{1}$ \\
\hline Evaluator agent & 0,297 & 0,309 & 0,210 & 0,281 & 0,207 & 0,355 & 0,226 & 0,343 & 0,239 & 0,302 \\
MLP & 0,677 & 0,669 & 0,489 & 0,507 & 0,513 & 0,806 & 0,530 & 0,696 & 0,506 & 0,485 \\
RBF & 1,009 & 0,833 & 0,656 & 0,985 & 0,878 & 0,959 & 0,620 & 0,831 & 0,643 & 0,783 \\
\hline
\end{tabular}

A Paired t-Test was applied to check that the difference between the methods can be considered as significative if a value $\alpha=0.05$ is established.

To evaluate the Advisor agent it is necessary to take into account that the aim of this agent is to detect inefficient tasks by means of gain functions, as explained in Section 3. The evaluation of the functioning of the Advisor agent was carried out by selecting those tasks with higher values for the gain function. The selected tasks were used to estimate the different scenarios for different execution values 
for the task. The estimation was performed using the values provided by the Evaluator agent, obtaining a concrete value for the task. In this way, Figure $2 \mathrm{a}$ presents the evolution of the system for the average status of 5 activities along 12 months. As shown, the evolution for the 5 activities can be considered as positive.
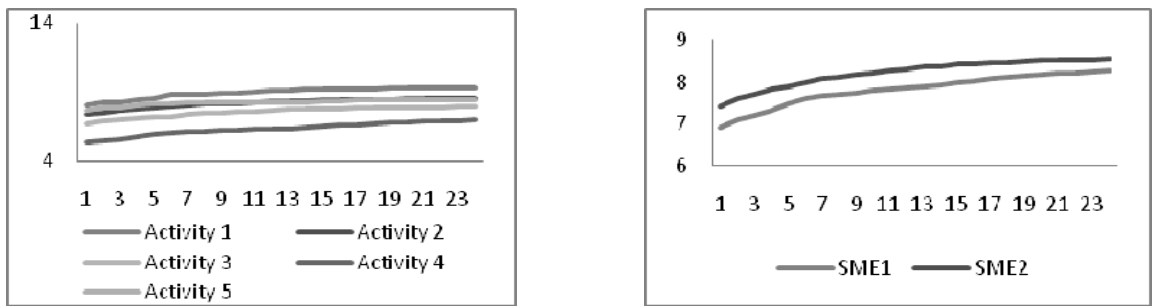

Fig. 2 a) Evolution of the average status of 5 activities during 12 months. b) Evolution of the average status of 2 SMEs during 12 months.

Looking at the evolution of the global efficiency for the activities analyses for two SMEs, shown in Figure 2b, it is possible to observe a growing tendency in the average status of the business along the time, which indicates a reduction of inefficient tasks in each of the activities. The results obtained demonstrate that the multiagent system caused a positive evolution in all enterprises. This evolution was reflected in the reduction of inefficient processes. The indicator used to determine the positive evolution of the companies was the state of each of the activities analyzed. After analyzing one of the company's activities, it was necessary to prove that the state of the activity (valued between 1 and 100) had increased beyond the state obtained in the previous three month period. The system considers small changes in the tasks performed in the SMEs, and all the experts that participated in the experiments considered 3 months as a significant time to evaluate the evolution of a SME related to these changes.

We had certain problems implementing the system, partly because the management and experts were not familiar with the use of computational devices and multiagent systems, so some courses were given to introduce them to these technologies and teach them how to use the system interface. The proposed novel multiagent system is a unique system useful for dynamic environments and open enough to be used in other enterprise environments. The experts noted that the behavior of the system improves as the number of cases in the memory of cases grows. This is a typical behavior in CBR-based systems. We found some difficulties with the surveys, because some of the SMEs were reticent to complete surveys.

The users indicated that the use of a web-based interface facilitates the use of the new system. However, some of them were reticent about trusting the system because they were reluctant to facilitate their internal data and because updating the information about the enterprise requires specialized human resources and time. However, the auditors and experts believe that the CBR-BDI agents may support their work and provide a highly appreciated decision support tool. They 
believe that this hybrid neural intelligent architecture has more advantages than disadvantages and that the system helped them to detect inconsistent processes in the enterprises. They tend to argue that the hybrid neural intelligent architecture should incorporate a shared memory of cases to compare data from different firms, but with the guarantee of data privacy.

As a conclusion, we can say that the use of innovative tools in business intelligence can notably help to detect potential risky situations and have a better understanding of their internal functioning. This article presented a multiagent-based tool aimed at modeling the behavior of SMEs and providing a decision support tool that can contribute to detect potential risky situations and to avoid them by acting on the tasks that compose each of the activities of the business.

\section{Acknowledgements}

This research has been partially supported by the project PET2008_0036 and FEDER funds.

\section{References}

Avogadri, R., Valentini, G.: Fuzzy ensemble clustering based on random projections for DNA microarray data analysis. Artificial Intelligence in Medicine 45(2-3), 173-183 (2009)

Bajo, J., De Paz, Y., De Paz, J.F., Corchado, J.M.: Integrating Case Planning and RPTW Neuronal Networks to Construct an Intelligent Environment for Health Care. Expert Systems with Applications 36(3), 5844-5858 (2009)

Baladandayuthapani, V., Ray, S., Mallick, B.K.: Bayesian Methods for DNA Microarray Data Analysis. Handbook of Statistics 25(1), 713-742 (2005)

Bianchia, D., Calogero, R., Tirozzi, B.: Kohonen neural networks and genetic classification. Mathematical and Computer Modelling 45(1-2), 34-60 (2007)

Borrajo, M.L., Corchado, J.M., Yáñez, J.C., Fdez-Riverola, F., Díaz, F.: Autonomous internal control system for small to medium firms. In: Muñoz-Ávila, H., Ricci, F. (eds.) ICCBR 2005. LNCS (LNAI), vol. 3620, pp. 106-121. Springer, Heidelberg (2005)

Calderon, T.G., Cheh, J.J.: A roadmap for future neural networks research in auditing and risk assessment. International Journal of Accounting Information Systems 3(4), $203-$ 236 (2002)

Chi-Jie, L., Tian-Shyug, L., Chih-Chou, C.: Financial time series forecasting using independent component analysis and support vector regression. Decision Support Systems 47(2), 115-125 (2009)

Colbert, J.L.: Risk. Internal Auditor, 36-40 (1995)

Committee of Sponsoring Organizations of the Treadway Commission (COSO), Guidance on Monitoring Internal Control Systems (COSO's Monitoring Guidance) (2009)

Corchado, J.M., Laza, R.: Constructing Deliberative Agents with Case-based Reasoning Technology. International Journal of Intelligent Systems 18(12), 1227-1241 (2003)

Corchado, J.M., Borrajo, M.L., Pellicer, M.A., Yanez, C.Y.: Neuro-symbolic system for business internal control. In: Perner, P. (ed.) ICDM 2004. LNCS (LNAI), vol. 3275, pp. 1-10. Springer, Heidelberg (2004) 
Dellaert, F.: The Expectation Maximization Algorithm, Technical Report. Georgia Institute of Technology (2002)

Ding, Y., Song, X., Zen, Y.: Forecasting financial condition of Chinese listed companies based on support vector machine. Expert Systems with Applications: An International Journal 34(4), 3081-3089 (2008)

Huang, S., Tsai, C., Yen, D.C., Cheng, Y.: A hybrid financial analysis model for business failure prediction. Expert Systems with Applications: An International Journal 35(3), 1034-1040 (2008)

Khashman, A.: A neural network model for credit risk evaluation. International Journal of Neural Systems 19(4), 285-294 (2009)

Kolodner, J.: Case-Based Reasoning. Morgan Kaufmann, San Francisco (1993)

Li, H., Sun, J.: Ranking-order case-based reasoning for financial distress prediction. Knowledge-Based Systems 21(8), 868-878 (2008)

$\mathrm{Li}, \mathrm{H}$., Sun, J.: Gaussian case-based reasoning for business failure prediction with empirical data in China. Information Sciences 179(1-2), 89-108 (2009)

Li, H., Sun, J.: On performance of case-based reasoning in Chinese business failure prediction from sensitivity, specificity, positive and negative values. Applied Soft Computing (in Press)

Li, H., Huang, H.B., Sun, J.: On sensitivity of case-based reasoning to optimal feature subsets in business failure prediction. Expert Systems with Applications (in Press)

Li, H., Liang, Y., Xu, Q.: Support vector machines and its applications in chemistry. Chemometrics and Intelligent Laboratory Systems 95(2), 188-198 (2009)

Lin, R., Wang, Y., Wu, C., Chuang, C.: Developing a business failure prediction model via RST, GRA and CBR. Expert Systems with Applications 36(2), 1593-1600 (2009)

Ramamoorti, S., Bailey, A.D., Traver, R.O.: Risk assessment in internal auditing: a neural network approach. Int. J. IntellSyst. Account Finance Manage (8), 159-180 (1999)

Risk and Insurance Management Society, Inc., Financial Crisis- A Wake up call for Enterprise Risk Management (2008)

Sawa, T., Ohno-Machado, L.: A neural network based similarity index for clustering DNA microarray data. Computers in Biology and Medicine 33(1), 1-15 (2003)

Sun, J., Li, H.: Data mining method for listed companies' financial distress prediction. Knowledge-Based Systems 21(1), 1-5 (2008a)

Sun, J., Li, H.: Listed companies' financial distress prediction based on weighted majority voting combination of multiple classifiers. Expert Systems with Applications 35(3), 818-827 (2008b)

Sun, J., Li, H.: Financial distress early warning based on group decision making. Computers \& Operations Research 36(3), 885-906 (2009a)

Sun, J., Li, H.: Financial distress prediction based on serial combination of multiple classifiers. Expert Systems with Applications 36(4), 8659-8666 (2009b) 